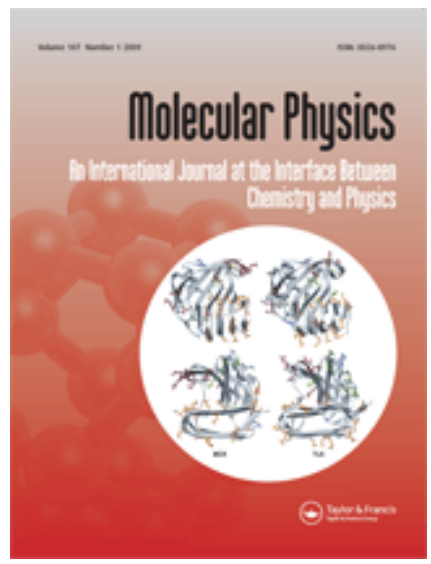

\title{
Anomalies in water as obtained from computer simulations of the TIP4P/2005 model: density maxima, and density, isothermal compressibility and heat capacity minima
}

\begin{tabular}{|c|c|}
\hline Journal: & Molecular Physics \\
\hline Manuscript ID: & TMPH-2008-0419.R1 \\
\hline Manuscript Type: & Special Issue Paper - Dr. Jean-Jacques Weis \\
\hline $\begin{array}{r}\text { Date Submitted by the } \\
\text { Author: }\end{array}$ & 14-Jan-2009 \\
\hline Complete List of Authors: & $\begin{array}{l}\text { Vega, Carlos; Univ.Complutense, Química Física } \\
\text { Pi, Helena; UCM, Quimica Fisica } \\
\text { Abascal, Jose Luis; UCM, Quimica Fisica } \\
\text { Noya, Eva; UCM, Quimica Fisica } \\
\text { McBride, Carl; UCM, Quimica Fisica; UCM, Quimica Fisica } \\
\text { Aragones, Juan; UCM, Quimica Fisica } \\
\text { Gonzalez, Miguel; UCM, Quimica Fisica }\end{array}$ \\
\hline Keywords: & $\begin{array}{l}\text { water anomalies, computer simulation, TIP4P/2005, Maximum in } \\
\text { density, Isothermal compressibility }\end{array}$ \\
\hline \multicolumn{2}{|c|}{$\begin{array}{l}\text { Note: The following files were submitted by the author for peer review, but cannot be converted } \\
\text { to PDF. You must view these files (e.g. movies) online. }\end{array}$} \\
\hline fig 1 & \\
\hline
\end{tabular}




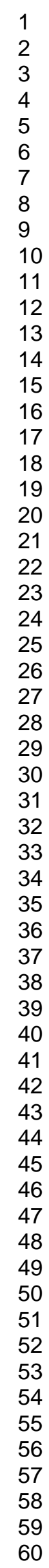

\section{$\$$ Manuscript Central}

URL: http://mc.manuscriptcentral.com/tandf/tmph 


\title{
Anomalies in water as obtained from computer simulations of the TIP4P/2005 model: density maxima, and density, isothermal compressibility and heat capacity minima
}

\author{
Helena L. Pi, Juan L. Aragones, Carlos Vega*, Eva G. Noya, \\ Jose L. F. Abascal, Miguel A. Gonzalez and Carl McBride \\ Departamento de Química Física, \\ Facultad de Ciencias Químicas, \\ Universidad Complutense de Madrid, \\ 28040 Madrid, Spain
}

(Dated: January 14, 2009)

\begin{abstract}
The so-called thermodynamic anomalies of water form an integral part of the peculiar behaviour of this both important and ubiquitous molecule. In this paper our aim is to establish whether the recently proposed TIP4P/2005 model is capable of reproducing a number of these anomalies. Using molecular dynamics simulations we investigate both the maximum in density and the minimum in the isothermal compressibility along a number of isobars. It is shown that the model correctly describes the decrease in the temperature of the density maximum with increasing pressure. At atmospheric pressure the model exhibits an additional minimum in density at a temperature of about 200K, in good agreement with recent experimental work on super-cooled confined water. The model also presents a minimum in the isothermal compressibility close to 310K. We have also investigated the atmospheric pressure isobar for three other water models; the SPC/E and TIP4P models also present a minimum in the isothermal compressibility, although at a considerably lower temperature than the experimental one. For the temperature range considered no such minimum is found for the TIP5P model.
\end{abstract}

Keywords:

* cvega@quim.ucm.es 


\section{INTRODUCTION}

The study of polar fluids has long been a topic of interest, even more-so since the development of perturbation theories and computer simulation techniques ${ }^{1,2}$. Amongst the many polar molecules, water stands out in particular. Water is a fascinating molecule, both from a practical and from a fundamental point of view. In the liquid phase water presents a number of anomalies when compared to other liquids ${ }^{3-7}$, whilst the solid phase exhibits a highly complex phase diagram, having at least fifteen different crystalline structures ${ }^{3,8}$. Due to its importance and its complexity, understanding the properties of water from a molecular point of view is of considerable interest and presents a veritable challenge.

Computer simulations of water started with the pioneering papers by Watts and Barker ${ }^{9}$ and by Rahman and Stillinger ${ }^{10}$ about forty years ago. However, a key issue that still exists when performing simulations of water is the choice of model for the pair potential that is used to describe the interaction between molecules ${ }^{1-15}$. The $\mathrm{SPC}^{16}, \mathrm{SPC} / \mathrm{E}^{17}, \mathrm{TIP} 3 \mathrm{P}^{18}$, TIP4P ${ }^{18}$ and TIP5P $\mathrm{P}^{19}$ models have become highly popular among the large community of people simulating water or water solutions. Each of the aforementioned models are rigid and non-polarisable, but naturally, real water is both flexible and can be polarised. It is almost needless to say that these models represent a very simplified version of the true interactions between real water molecules. Water also exhibits important quantum nuclear effects, so a realistic description of water should also take this into account ${ }^{20}$. That said, it is of interest at this stage to analyse how far it is possible to go in describing real water using these simple models.

In recent years the inexorable increase in computing power has permitted the calculation of properties that were previously inaccessible to simulations. These properties can be used as new 'target quantities' when fitting the parameters for any new potential. More importantly, some of these properties have provided stringent tests for the existing water models. In particular, some of the authors have recently determined the phase diagram for different water models and have found that the performance of the models can vary significantly ${ }^{21-25}$. For example, it has been found that TIP4P provides a qualitatively correct description of the global phase diagram of water, whereas the SPC, SPC/E, and TIP5P models do not. ${ }^{26}$ For the SPC and SPC/E models the melting temperature and the maximum in density of water occur at temperatures far below the experimental values. Taking this into 
account some of the authors proposed a new rigid non-polarisable model with the following constraints: that it should be based on the TIP4P model, since this model provides a reasonable description of the phase diagram of water. It should also reproduce the maximum in density of liquid water (notice that most of models fail when it comes to predicting the location of the density maximum ${ }^{27}$ ). Finally, the model should account for the vaporisation enthalpy of real water, but only after incorporating the self-polarisation energy correction proposed by Berendsen et al. for the $\mathrm{SPC} / \mathrm{E}$ model ${ }^{17}$. It was from these considerations that the TIP4P/2005 model of water arose. ${ }^{25}$ In the paper in which the model was presented it was shown that TIP4P/2005 correctly describes the global phase diagram of water, the diffusion coefficient at atmospheric pressure and temperature, the maximum in density along the atmospheric pressure isobar, the density of the ice polymorphs, and the equation of state of liquid water at high pressures. Afterwards, additional studies have shown that the model is also able to provide a good description of the vapour-liquid equilibria ${ }^{28}$ and the surface tension ${ }^{29}$. Further information concerning the performance of TIP4P/2005 and its comparison with other water models can be found elsewhere ${ }^{26}$. The overall results indicate that TIP4P/2005 is probably close to being the best rigid non-polarisable model that can be achieved; any significant improvement would require the introduction of 'new' physics, i.e., flexibility, polarisability and nuclear quantum effects.

In this paper we are interested in the thermodynamic response functions in the region where water exhibits 'anomalous' behaviour. In particular, these are the expansion coefficient (which vanishes at temperatures close to the melting point, resulting in the well known maximum of density at about $4^{\circ} \mathrm{C}$ at atmospheric pressure) and the isothermal compressibility $^{30}\left(\kappa_{T}\right)$ which shows a minimum at $46.5^{\circ} \mathrm{C}$ at $p=1$ bar. Moreover, we shall investigate whether the model is able to describe the pressure dependence of these thermodynamic properties, namely, the decrease in the temperature of the density maximum and the shift towards slightly higher temperatures of the minimum in $\kappa_{T}$ as the pressure increases. It will be shown that the model is indeed able to describe these two features quite well, which provides further evidence of the robust behaviour of the model even when estimating properties that were not taken into account in the original fitting process. 


\section{METHODOLOGY}

The interaction between water molecules will be described by the TIP4P/2005 model. ${ }^{25}$ In this model, a Lennard-Jones centre is located on the oxygen atom, positive charges are placed on the hydrogen atoms and a negative charge is located at the site $M$ situated along the H-O-H bisector. For the simulations we have used the molecular dynamics package GROMACS (version 3.3). ${ }^{31}$ The Lennard-Jones potential has been truncated at $9.0 \AA$. Long range corrections were applied to the Lennard-Jones part of the potential (for both the energy and pressure). Ewald summations were used to deal with electrostatic contributions. The real part of the Coulombic potential was truncated at $9.0 \AA$. The Fourier component of the Ewald sums was evaluated by using the Particle Mesh Ewald (PME) method of Essmann et al. ${ }^{32}$ The width of the mesh was $1 \AA$ and a fourth degree polynomial was used. The simulation box was cubic throughout the whole simulation and the geometry of the water molecules was enforced using constraints. ${ }^{33,34}$ The temperature was set by using a Nosé-Hoover ${ }^{35,36}$ thermostat with a relaxation time of 2 ps. To maintain constant pressure an isotropic Parrinello-Rahman barostat ${ }^{37,38}$ with a relaxation time of 2 ps was used. As a check, at two pressures, $p=1$ bar and $p=400$ bar, Monte Carlo simulations were performed using a bespoke program. The Monte Carlo densities were in complete agreement with those obtained from molecular dynamics using GROMACS.

To determine the maximum in density, molecular dynamics simulations have been performed along the isobars $p=1,400,1000$, and 1500 bar. The number of molecules used in the simulations was 256. Long runs are required to determine the maximum in density; for each thermodynamic state twenty million time-steps were performed. Since the time step was $2 \mathrm{fs}$, the results presented here are an average of the properties of the system obtained from runs of $40 \mathrm{~ns}$. The simulations were started at high temperatures, and the final configuration of a particular run was used as the initial configuration for a lower temperature simulation. Typically about 6 to 8 different temperatures were studied along each isobar. The isothermal compressibility is defined as:

$$
\kappa_{T}=-\frac{1}{V}\left(\frac{\partial V}{\partial p}\right)_{T} .
$$

The literature for the isothermal compressibility of water models is rather scant ${ }^{39,40}$. The computational overhead required for a study of the variation of $\kappa_{T}$ with temperature with 
sufficient accuracy has, until now, been prohibitive. Here we have evaluated the isothermal compressibility for the following water models: SPC/E ${ }^{17}$, TIP4P ${ }^{18}$, TIP4P $/ 2005^{25}$ and TIP5P ${ }^{19}$ along the atmospheric pressure isobar. The isothermal compressibility has been evaluated using the well known fluctuation formula

$$
\kappa_{T}=\frac{\left\langle V^{2}\right\rangle-\langle V\rangle^{2}}{k T\langle V\rangle} .
$$

The volume fluctuations were typically averaged over 20 ns using a time-step of 1 fs. Preliminary results for TIP4P/2005 model were sufficiently close to the experimental value as to warrant a more precise calculation. Thus, for this model, the isothermal compressibility has been determined via two different procedures using, in both cases, a time-step of $0.5 \mathrm{fs}$ and a sample size of 500 molecules. The first method is the application of the volume fluctuation formula, typically over 40 million molecular dynamics time-steps, for a total simulation length of about 20ns. The relative uncertainty of the calculated compressibilities is about $\pm 3 \%$. The second route is the numerical evaluation of the derivative appearing in the definition of $\kappa_{T}$ (Eq. 1). To this end, the results for the equation of state of five different state points (at different pressures but at the same temperature) were fitted to a second degree polynomial. In addition to the state point of interest, for which the volume is already known from the runs used to calculate $\kappa_{T}$ via the fluctuation route, four additional runs are required: two runs at the same temperature but at pressures of 200 and 400 bar higher, and another two runs at pressures 200 and 400 bar lower. The simulation length of these additional runs was $6.5 \mathrm{~ns}$, so in the end the computational cost of both routes is almost the same. We have also computed the heat capacity at constant pressure $C_{p}$ for the TIP4P/2005 model. $C_{p}$ is defined as :

$$
C_{p}=\left(\frac{\partial H}{\partial T}\right)_{p} .
$$

Thus in order to compute $C_{p}$ the enthalpy at each temperature was first calculated. These values for the enthalpy were then fitted to a polynomial and this fit was then differentiated with respect to temperature to obtain $C_{p}(T)$.

\section{RESULTS}

The simulation results for the densities are reported in Tables I, II, III and IV for $p=1$, 400, 1000 and 1500 isobars, respectively. For the four isobars considered a clear maximum 
in density has been found. The results for $p=1$ bar and $p=400$ bar, along with experimental measurements, are plotted in Fig. 1. It can be seen that the model is able to reproduce the experimental data quite nicely. The density maximum for each isobar was obtained by fitting the densities around the maximum (typically 5 or 6 densities were used in the fit) to a second or a third degree polynomial. The values of the maximum are reported in Table V. In Fig. 2 the temperatures at which the maxima appear (TMD) are compared to the experimental values ${ }^{41}$. As can be seen, the agreement is rather good. The model correctly predicts a decrease of about $33 \mathrm{~K}$ in the temperature of the maximum when going from atmospheric pressure to a pressure of about 1500 bar. In other words, 45 bar are required to induce a decrease in the TMD of about one degree. For $\mathrm{D}_{2} \mathrm{O}$ Angell and Kanno found a similar lowering of the TMD with pressure. ${ }^{42}$ For the TIP4P/2005 not only does the TMD decrease with pressure but the melting temperature does too. In Fig.2 the melting curve of ice $\mathrm{I}_{h}$ (taken from our previous work ${ }^{25,43,44}$ ) is plotted along the TMD curve determined in this work. As can be seen, both curves have a negative slope. Notice also that at room pressure the distance between the melting curve of ice $\mathrm{I}_{h}$ and the TMD is about $25 \mathrm{~K}$, which is in contrast with the $4 \mathrm{~K}$ of difference found experimentally. The impossibility of reproducing simultaneously both the TMD and the melting temperature in models having three charges has been discussed previously ${ }^{26,27}$.

Another interesting issue is the behaviour of the density along the atmospheric pressure isotherm at very low temperatures. Experimentally, it is not possible to obtain the density of water at temperatures below $233 \mathrm{~K}$ (the homogeneous stability limit of water at atmospheric pressure $^{45}$ ) due to spontaneous nucleation and freezing. However, recently, it has been possible to avoid the formation of ice by confining water in pores a few nanometers in diameter (most probably because of the decrease in the freezing point of water due to confinement as described qualitatively by the Gibbs-Thomson approach ${ }^{46}$ ). Thanks to this the density of deuterated water has recently been determined for the first time ${ }^{47}$ for temperatures as low as $160 \mathrm{~K}$, reporting the existence of a minimum in the density at a temperature of about 200 K. A similar study (for water instead of deuterated water) was performed by Mallamace et $a l .{ }^{48}$. By using infra-red (IR) spectroscopy, it was possible to determine the density of liquid water in the super-cooled regime down to $150 \mathrm{~K}$. In Fig. 3 the reported experimental densities (from IR spectroscopy of a sample within a $1.5 \mathrm{~nm}$ pore) for liquid water along the atmospheric pressure isobar are compared to those obtained in this work from molecular 
dynamics simulations of the TIP4P/2005 water model. The agreement is surprisingly good, and the location of the minimum around $200 \mathrm{~K}$ is described properly by TIP4P/2005. Such a minimum in density has also been found ${ }^{49,50}$ in computer simulations of the TIP5P and ST2 models of water, but the agreement with experiment was not as good as that exhibited by TIP4P/2005. For temperatures below this minimum the density increases as the temperature decreases (as in a normal fluid). In Fig. 4 we compare the equation of state of supercooled water to the equation of state of ice $\mathrm{I}_{h}$ for the TIP4P/2005 model along the room pressure isobar. ${ }^{51}$ The minimum in density of supercooled water occurs just when the density is approaching that of the ice $\mathrm{I}_{h}$. However, we did not succeed in obtaining ice $\mathrm{I}_{h}$ by cooling water; the radial distribution functions of the supercooled water (at room pressure) below $200 \mathrm{~K}$ are clearly different from those of ice $\mathrm{I}_{h}$. Rather the minimum in density corresponds to the formation of a glassy state. Note that the existence of such a minimum is not only restricted to water but is also present in materials such as tellurium ${ }^{52}$. Interestingly the melting curve for tellurium exhibits re-entrance. Such re-entrant behaviour was also found in our studies of the phase diagram of water models ${ }^{21}$.

We have also computed the self-diffusion coefficient at $p=1$ bar and $p=1000$ bar. The results, presented in Fig. 5, show that the diffusion coefficient drops significantly as the temperature decreases. The decay is less pronounced at higher pressures. At low temperatures the diffusion coefficient increases significantly with pressure (this behaviour is anomalous ${ }^{30}$ since, for most fluids, it decreases with increasing pressure). One can imagine that the application of pressure somehow breaks the hydrogen bond network, thus aiding diffusion processes. Some indirect evidence of the decrease in hydrogen bonding with pressure can be obtained from the analysis of the different contributions (Lennard-Jones and Coulombic) to the residual internal energy. In Table VI the different contributions to the residual internal energy obtained from molecular dynamics of the TIP4P/2005 model along the $\mathrm{T}=224.6 \mathrm{~K}$ isotherm (at different pressures) are given. Notice the large decrease (in absolute value) of the Coulombic energy with pressure, clearly pointing to a reduction of hydrogen bonding with pressure. The Lennard-Jones contribution is more repulsive at low pressures (even though the density is lower) as a consequence of the stronger hydrogen bond found at lower pressures. At temperatures above $280 \mathrm{~K}$ the diffusion coefficients at the two pressures considered become virtually identical as the differences fall to within the statistical uncertainty. The agreement with the experimental values of the diffusion coefficient ${ }^{53}$ is quite good. 
Let us now focus on another of the 'anomalous' properties of water, the isothermal compressibility. The experimental measurements ${ }^{54-57}$ show that, at atmospheric pressure, $\kappa_{T}$ drops as the temperature increases from the melting temperature up to $46.5^{\circ}$. Above this temperature water behaves as a normal liquid and the isothermal compressibility increases with temperature. It is also well established from experiment that the temperature for which $\kappa_{T}$ is minimal shifts slightly towards higher values as the pressure increases. Contrary to the maximum in density, the ability of the water models to account for the compressibility minimum has not yet been established. As mentioned in the previous section, simulation studies of the isothermal compressibility are few and far between ${ }^{39,40}$. Moreover, the computational resources available did not allow the extended simulations needed to calculate $\kappa_{T}$ with the precision required to determine whether the most common water models predict the compressibility minimum. For this reason we have calculated the compressibility for a select few 'popular' water models. The results are presented in Table VII for the SPC/E, TIP4P and TIP5P models and in Table VIII for TIP4P/2005. The calculations for SPC/E, TIP4P and TIP5P were performed at atmospheric pressure for three different temperatures, namely $260 \mathrm{~K}, 300 \mathrm{~K}$ and $360 \mathrm{~K}$. Fig. 6a shows that the temperature dependence of the isothermal compressibility for TIP5P does not follow the experimental pattern since, for this model, $\kappa_{T}$ is a monotonously increasing function along the whole experimental liquid range. Despite the fact that the slope of the TIP5P curve is opposite to the experimental one, the values of $\kappa_{T}$ are coincident in a narrow range of temperatures because the simulation and the experimental curves cross at a temperature close to the freezing point of liquid water. For temperatures near the boiling point the TIP5P model fails completely, where the predicted compressibility at $360 \mathrm{~K}$ is almost twice the experimental value.

As for the performance of the SPC/E and TIP4P models, both provide fairly similar results. In fact their curves are parallel, showing a more or less defined minimum around $270 \mathrm{~K}$ (more computations would be needed to determine the precise location of the minima). The results of SPC/E are somewhat shifted to higher temperatures with respect to those of TIP4P, which results in a slightly better agreement with the experimental data. At high temperatures, the differences between simulation and experiment are quite noticeable for both SPC/E and TIP4P (though less dramatic than in the TIP5P case).

As can be seen in Fig. 6b, the TIP4P/2005 model provides an excellent description of the isothermal compressibility of water. The compressibilities obtained from the two routes 
(differenciation of the equation of state and fluctuation formula) were found to be mutually consistent. The departures of the calculated compressibilities with respect to the experimental values ${ }^{54-57}$ are in general smaller than $7 \%$. By fitting the TIP4P/2005 compressibilities at atmospheric pressure to a third degree polynomial, a minimum in the isothermal compressibility is found for a temperature of about $37^{\circ} \mathrm{C}$ which is in good agreement with the experimental value $\left(46.5^{\circ} \mathrm{C}\right)$. Thus the model is able to accurately reproduce not only the isothermal compressibility along the whole liquid range at atmospheric pressure but also the compressibility minimum. This assertion is particularly true when one compares the TIP4P/2005 predictions with those of the other models, shown in Fig 6 (notice that we have plotted both panels using the same scale). In Fig 6, we also present the compressibility results at a higher pressure (1000 bar). TIP4P/2005 predictions for this isobar are slightly better than those for atmospheric pressure. A compressibility minimum is also found at 1000 bar, and, in accordance with the experiment, the minimum appears at an slightly higher temperature than it does at atmospheric pressure ${ }^{54}$.

Finally we have examined the behaviour of the heat capacity at constant pressure for two isobars, namely, $p=1$ bar and $p=1000$ bar. The results are presented in figure 7 . As can be seen, the model hints at the existence of minima in the heat capacity for both of these isobars. The location of the minimum seems to move to lower temperatures as the pressure increases, in concordance with experiment (see fig.7 of Ref. ${ }^{41}$ ).

In figure 8 the values of $C_{p}$ from the simulations are compared to the experimental ${ }^{58}$ values for water and $\mathrm{D}_{2} \mathrm{O}$. As one can see, the model overestimates the experimental values of the heat capacity of water at constant pressure. The large difference in the experimental values of the heat capacity of $\mathrm{H}_{2} \mathrm{O}$ and $\mathrm{D}_{2} \mathrm{O}$ demonstrate the importance of nuclear quantum effects in the description of the heat capacity of water. Not surprisingly, the values of TIP4P/2005 which were obtained through classical simulations lie closer to the experimental values of $\mathrm{D}_{2} \mathrm{O}$, probably reflecting the somewhat more classical behaviour of $\mathrm{D}_{2} \mathrm{O}$ with respect to $\mathrm{H}_{2} \mathrm{O}$.

Taking into account the success of TIP4P/2005 in describing most of the properties of water, the failure in the quantitative description of $C_{p}$ (along with the important differences in the experimental values of $\mathrm{D}_{2} \mathrm{O}$ and $\mathrm{H}_{2} \mathrm{O}$ ) points out the necessity of incorporating nuclear quantum effects for a quantitative description of this property. Although one could incorporate quantum corrections empirically ${ }^{25,59}$, quantum simulations (for example, via path 
integrals) are probably the only way to correctly describe the heat capacity of water ${ }^{60,61}$.

\section{CONCLUSIONS}

In this work molecular dynamics simulations have been undertaken for liquid water along several isobars. The TIP4P/2005 model has been used to describe the interaction between water molecules. The results obtained in relatively long runs (i.e., $40 \mathrm{~ns}$ ) indicate that a maximum in density is found for the isobars considered in this work (i.e., $p=1,400,1000$ and 1500 bar). The TMD decreases by about $1 \mathrm{~K}$ for each 45 bar of applied pressure. Thus, the TIP4P/2005 model, designed to reproduce the TMD at atmospheric pressure, is also able to predict the dependence of the TMD with pressure. Motivated by recent experimental work the behaviour of the density at low temperatures along the atmospheric pressure isobar was also studied, resulting in a density minimum at temperatures around $200 \mathrm{~K}$. The location and density at the minimum of the model are in very nice agreement with recent experimental work on the equation state of water at very low temperatures (obtained by confining water in narrow pores in order to prevent the nucleation of ice). Also in agreement with experimental measurements we have found a significant increase of the diffusion coefficient of super-cooled water with pressure.

Finally, we have also computed the isothermal compressibility along the atmospheric pressure isobar for several water models and, once again, the results for TIP4P/2005 are those closest to experimental values. A minimum in the isothermal compressibility was found for temperatures around $37^{\circ} \mathrm{C}$, once again in good agreement with the location of the minimum found in experiments $\left(46.5^{\circ} \mathrm{C}\right)$. Not only is the location of the minimum well described by the model, but the value at the minimum as well. Finally, we have calculated $\kappa_{T}$ at several temperatures at a higher pressure (1000 bar) observing that TIP4P/2005 also exhibits a compressibility minimum. In accordance with experiment, the minimum is shifted towards slightly higher temperatures ${ }^{62}$ when the pressure increases from 1 bar to 1000 bar.

The results of this work indicate that the TIP4P/2005 is able to reproduce almost quantitatively many of the anomalous properties of water occurring at low temperatures with the exception of the heat capacity. Therefore, the model can be used with confidence to obtain a better understanding of the behaviour of water in the super-cooled regime where the experimental determination of properties is a difficult task. It seems that for super-cooled 
water the performance of the TIP4P/2005 model of water supersedes the performance of other more traditional models.

\section{Acknowledgments}

This work has been funded by grants FIS2007-66079-C02-01 from the DGI (Spain), S0505/ESP/0299 from the CAM, and 910570 from the UCM.

1 L. Verlet and J. J. Weis, Molec. Phys. 28, 665 (1974).

2 D. Levesque and J. J. Weis, Phys. Rev. E 49, 5131 (1994).

3 D. Eisenberg and W. Kauzmann, The Structure and Properties of Water (Oxford University Press, London, 1969).

4 V. F. Petrenko and R. W. Whitworth, Physics of Ice (Oxford University Press, 1999).

5 J. L. Finney, Phil.Trans.R.Soc.Lond.B 359, 1145 (2004).

6 M. Chaplin, http://www.lsbu.ac.uk/water/ (2005).

7 J. L. Finney, J. Molec. Liq. 90, 303 (2001).

8 C. G. Salzmann, P. G. Radaelli, A. Hallbrucker, E. Mayer, and J. L. Finney, Science 311, 1758 (2006).

9 J. A. Barker and R. O. Watts, Chem. Phys. Lett. 3, 144 (1969).

10 A. Rahman and F. H. Stillinger, J. Chem. Phys. 55, 3336 (1971).

11 R. M. Lynden-Bell, J. C. Rasaiah, and J. P. Noworyta, Pure Appl. Chem. 73, 1721 (2001).

12 K. M. Åberg, A. P. Lyubartsev, S. P. Jacobsson, and A. Laaksonen, J. Chem. Phys. 120, 3770 (2004).

13 M. Ferrario, G. Ciccotti, E. Spohr, T. Cartailler, and P.Turq, J. Chem. Phys. 117, 4947 (2002).

14 D. Paschek, J. Chem. Phys. 120, 6674 (2004).

15 C. R. W. Guimaraes, G. Barreiro, C. A. F. de Oliveria, and R. B. de Alencastro, Brazil. J. Phys. 34, 126 (2004).

16 H. J. C. Berendsen, J. P. M. Postma, W. F. van Gunsteren, and J. Hermans, Intermolecular Forces, ed. B. Pullman, page 331 (Reidel, Dordrecht, 1982).

17 H. J. C. Berendsen, J. R. Grigera, and T. P. Straatsma, J. Phys. Chem. 91, 6269 (1987). 
18 W. L. Jorgensen, J. Chandrasekhar, J. D. Madura, R. W. Impey, and M. L. Klein, J. Chem. Phys. 79, 926 (1983).

19 M. W. Mahoney and W. L. Jorgensen, J. Chem. Phys. 112, 8910 (2000).

20 B. Guillot, J. Molec. Liq. 101, 219 (2002).

21 E. Sanz, C. Vega, J. L. F. Abascal, and L. G. MacDowell, Phys. Rev. Lett. 92, 255701 (2004).

22 C. Vega, J. L. F. Abascal, E. Sanz, L. G. MacDowell, and C. McBride, J. Phys. Cond. Mat. 17, S3283 (2005).

23 C. Vega, E. Sanz, J. L. F. Abascal, and E. G. Noya, J. Phys. Condens. Matter 20, 153101 (2008).

24 J. L. F. Abascal, E. Sanz, R. G. Fernández, and C. Vega, J. Chem. Phys. 122, 234511 (2005).

25 J. L. F. Abascal and C. Vega, J. Chem. Phys. 123, 234505 (2005).

26 C. Vega, J. L. F. Abascal, M. M. Conde, and J. L. Aragones, Faraday Discussions 141, 251 (2009).

27 C. Vega and J. L. F. Abascal, J. Chem. Phys. 123, 144504 (2005).

28 C. Vega, J. L. F. Abascal, and I. Nezbeda, J. Chem. Phys. 125, 034503 (2006).

29 C. Vega and E. de Miguel, J. Chem. Phys. 126, 154707 (2007).

30 P. G. Debenedetti, J. Phys. Cond. Mat. 15, R1669 (2003).

31 D. V. der Spoel, E. Lindahl, B. Hess, G. Groenhof, A. E. Mark, and H. J. C. Berendsen, J. Comput. Chem. 26, 1701 (2005).

32 U. Essmann, L. Perera, M. L. Berkowitz, T. Darden, H. Lee, and L. G. Pedersen, J. Chem. Phys. 103, 8577 (1995).

33 J. P. Ryckaert, G. Ciccotti, and H. J. C. Berendsen, J.Comp.Phys. 23, 327 (1977).

34 H. J. C. Berendsen and W. F. van Gunsteren, in Molecular Liquids-Dynamics and Interactions (Reidel Dordretch, 1984), Proceedings of the NATO Advanced Study Institute on Molecular Liquids, pp. 475-500.

35 S. Nosé, Molec. Phys. 52, 255 (1984).

36 W. G. Hoover, Phys. Rev. A 31 (1985).

37 M. Parrinello and A. Rahman, J. Appl. Phys. 52, 7182 (1981).

38 S. Nosé and M. L. Klein, Molec. Phys. 50, 1055 (1983).

39 K. A. Motakabbir and M. Berkowitz, J. Phys. Chem. 94, 8359 (1990).

40 A. Glättli, X. Daura, and W. van Gunsteren, J. Chem. Phys. 116, 9811 (2002). 
41 R. A. Fine and F. J. Millero, J. Chem. Phys. 63, 89 (1975).

42 C. A. Angell and H. Kanno, Science 193, 1121 (1976).

43 J. L. F. Abascal, E. Sanz, and C. Vega, Phys.Chem.Chem.Phys. 11, 556 (2009).

44 J. L. Aragones, M. M. Conde, E. G. Noya, and C. Vega, Phys.Chem.Chem.Phys. 11, 543 (2009).

45 H. Kanno, R. J. Speedy, and C. A. Angell, Science 189, 880 (1975).

46 S. Kittaka, S. Ishimaru, M. Kuranishi, T. Matsuda, and T. Yamaguchi, Phys. Chem. Chem. Phys. 8, 3223 (2006).

47 D. Liu, Y. Zhang, C. C. Cheng, C. Y. Mou, P. H. Poole, and S. H. Chen, Proc. Natl. Acad. Sci. 104, 9570 (2007).

48 F. Mallamace, C. Branca, M. Broccio, C. Corsaro, C. Y. Mou, and S. H. Chen, Proc. Natl. Acad. Sci. 104, 18387 (2007).

49 D. Paschek, Phys.Rev.Lett. 94, 217802 (2005).

50 P. H. Poole, I. Saika-Voivod, and F. Sciortino, J.Phys.Condens.Matter 17, L431 (2005).

51 E. G. Noya, C. Menduina, J. L. Aragonés, and C. Vega, J. Phys. Chem. C 111, 15877 (2007).

52 C. A. Angell, Nature Nanotechnology 2, 396 (2007).

53 R. F.X Prielmeier, E.W.Lang and H.D.Ln, Ber.Bunsenges.Phys.Chem 92, 1111 (1988).

54 A. Saul and W. Wagner, J. Phys. Chem. Ref. Data 18, 1537 (1989).

55 G. S. Kell, J. Chem. Eng. Data 20, 97 (975).

56 R. J. Speedy and C. A. Angell, J. Chem. Phys. 65, 851 (1976).

57 T. S. Carlton, J. Phys. Chem. B 111, 13398 (2007).

58 C. A. Angell, M. Oguni, and W. J. Sichina, J.Phys.Chem. 86, 998 (1982).

59 H. W. Horn, W. C. Swope, J. W. Pitera, J. D. Madura, T. J. Dick, G. L. Hura, and T. HeadGordon, J. Chem. Phys. 120, 9665 (2004).

60 W. Shinoda and M. Shiga, Phys. Rev. E 71, 041204 (2005).

61 G. S. D. Buono, P. J. Rossky, and J. Schnitker, J. Chem. Phys. 95, 3728 (1991).

62 R. A. Fine and F. J. Millero, J. Chem. Phys. 59, 5529 (1973). 
TABLE I: Molecular dynamics results for the TIP4P/2005 model of water along the $p=1$ bar isobar. Only the residual part of the internal energy is given.

\begin{tabular}{cccc}
\hline \hline $\mathrm{T} / \mathrm{K}$ & $\mathrm{p} / \mathrm{bar}$ & $\rho /\left(\mathrm{g} / \mathrm{cm}^{3}\right)$ & $\mathrm{U} /(\mathrm{kJ} / \mathrm{mol})$ \\
\hline 150.0 & 1 & 0.9379 & -57.05 \\
156.0 & 1 & 0.9370 & -56.87 \\
162.4 & 1 & 0.9359 & -56.68 \\
169.0 & 1 & 0.9347 & -56.47 \\
176.0 & 1 & 0.9341 & -56.25 \\
183.3 & 1 & 0.9331 & -55.99 \\
191.0 & 1 & 0.9341 & -55.74 \\
199.0 & 1 & 0.9339 & -55.51 \\
207.3 & 1 & 0.9432 & -54.81 \\
215.8 & 1 & 0.9559 & -54.04 \\
224.6 & 1 & 0.9659 & -53.27 \\
233.5 & 1 & 0.9787 & -52.47 \\
242.7 & 1 & 0.9890 & -51.70 \\
252.1 & 1 & 0.9953 & -50.98 \\
261.9 & 1 & 0.9991 & -50.28 \\
272.2 & 1 & 1.0008 & -49.57 \\
283.0 & 1 & 1.0002 & -48.84 \\
294.4 & 1 & 0.9987 & -48.10 \\
300.0 & 1 & 0.9972 & -47.73 \\
\hline \hline
\end{tabular}


TABLE II: Molecular dynamics results for the TIP4P/2005 model of water along the $p=400$ bar isobar. Only the residual part of the internal energy is given.

\begin{tabular}{|c|c|c|c|}
\hline \multicolumn{3}{|c|}{$\mathrm{T} / \mathrm{K} \mathrm{p} / \mathrm{bar} \rho /\left(\mathrm{g} / \mathrm{cm}^{3}\right)$} & $\mathrm{J} /(\mathrm{kJ} / \mathrm{mol})$ \\
\hline 215.8 & 400 & 0.9766 & -53.39 \\
\hline 224.6 & 400 & 0.9963 & -52.92 \\
\hline 233.5 & 400 & 1.0051 & -52.27 \\
\hline 242.7 & 400 & 1.0125 & -51.57 \\
\hline 252.1 & 400 & 1.0171 & -50.90 \\
\hline 261.9 & 400 & 1.0192 & -50.24 \\
\hline 272.2 & 400 & 1.0200 & -49.55 \\
\hline 283.0 & 400 & 1.0190 & -48.85 \\
\hline 294.4 & 400 & 1.0164 & -48.14 \\
\hline
\end{tabular}

TABLE III: Molecular dynamics results for the TIP4P/2005 model of water along the $p=1000$ bar isobar. Only the residual part of the internal energy is given.

\begin{tabular}{|c|c|c|c|}
\hline \multicolumn{3}{|c|}{$\mathrm{T} / \mathrm{K} \mathrm{p} / \mathrm{bar} \rho /\left(\mathrm{g} / \mathrm{cm}^{3}\right.$} & \multirow{2}{*}{$\frac{\mathrm{J} /(\mathrm{kJ} / \mathrm{mol})}{-53.25}$} \\
\hline 215.8 & 1000 & 1.0308 & \\
\hline 224.6 & 1000 & 1.0397 & -52.62 \\
\hline 233.5 & 1000 & 1.0438 & -52.04 \\
\hline 242.7 & 1000 & 1.0463 & -51.43 \\
\hline 252.1 & 1000 & 1.0476 & -50.82 \\
\hline 261.9 & 1000 & 1.0475 & -50.19 \\
\hline 272.2 & 1000 & 1.0462 & -49.55 \\
\hline 283.0 & 1000 & 1.0441 & -48.89 \\
\hline 294.4 & 1000 & 1.0407 & -48.20 \\
\hline
\end{tabular}


TABLE IV: Molecular dynamics results for the TIP4P/2005 model of water along the $p=1500$ bar isobar. Only the residual part of the internal energy is given.

\begin{tabular}{|c|c|c|c|}
\hline \multicolumn{3}{|c|}{$\mathrm{T} / \mathrm{K} \mathrm{p} / \mathrm{bar} \rho /\left(\mathrm{g} / \mathrm{cm}^{3}\right)$} & \multirow{2}{*}{$\frac{\mathrm{U} /(\mathrm{kJ} / \mathrm{mol})}{-54.25}$} \\
\hline 199.0 & 1500 & 1.0472 & \\
\hline 207.3 & 1500 & 1.0617 & -53.61 \\
\hline 215.8 & 1500 & 1.0667 & -53.06 \\
\hline 224.6 & 1500 & 1.0678 & -52.52 \\
\hline 233.5 & 1500 & 1.0702 & -51.94 \\
\hline 242.7 & 1500 & 1.0701 & -51.37 \\
\hline 252.1 & 1500 & 1.0700 & -50.78 \\
\hline 261.9 & 1500 & 1.0682 & -50.18 \\
\hline 272.2 & 1500 & 1.0662 & -49.56 \\
\hline 283.0 & 1500 & 1.0631 & -48.91 \\
\hline 294.4 & 1500 & 1.0593 & -48.24 \\
\hline
\end{tabular}

TABLE V: Temperature of maximum density at different pressures. The TMD has been obtained by fitting the data in the proximity of the maximum to a quadratic or a cubic polynomial. The temperature ranges used in the fit were $260-300 \mathrm{~K}$ for $p=1$ bar, $242-295 \mathrm{~K}$ for $p=400$ bar, 233-283 K for $p=1000$ bar, and 207-272 K for $p=1500$ bar.

\begin{tabular}{ccccc}
\hline \hline $\mathrm{p} / \mathrm{bar}$ & 1 & 400 & 1000 & 1500 \\
$\mathrm{TMD} / \mathrm{K}$ & $277(3)$ & $270(3)$ & $256(3)$ & $244(3)$ \\
\hline \hline
\end{tabular}


TABLE VI: Different contributions to the residual internal energy of water along the $T=224.6 K$ isotherm. Results were obtained from molecular dynamics simulations of the TIP4P/2005 model of water. The Lennard-Jones contribution $U_{\text {Lennard-Jones }}$, the Coulombic contribution $U_{\text {Coulombic }}$ and the total residual energy $U$ are reported. All energies are given in $\mathrm{kJ} / \mathrm{mol}$.

\begin{tabular}{|c|c|c|c|}
\hline \multicolumn{2}{|c|}{$\mathrm{p} /$ bar $U_{\text {Lennard-Jones }}$} & \multirow{2}{*}{$\begin{array}{c}U_{\text {Coulombic }} \\
-64.61\end{array}$} & \multirow{2}{*}{$\begin{array}{c}U \\
-53.27\end{array}$} \\
\hline 1 & 11.34 & & \\
\hline 400 & 11.08 & -64.00 & -52.92 \\
\hline 1000 & 10.78 & -63.40 & -52.62 \\
\hline 1500 & 10.66 & -63.18 & -52.52 \\
\hline
\end{tabular}

TABLE VII: Isothermal compressibility $\kappa_{T}$ for $p=1$ bar as obtained in this work for the TIP4P, SPC/E and TIP5P models of water. Reported values correspond to $\left(\kappa_{T} / \mathrm{bar}^{-1}\right) \times 10^{6}$.

\begin{tabular}{ccccc}
\hline \hline T/K & \multicolumn{4}{c}{ TIP4P SPC/E TIP5P Experiment } \\
260 & 51.8 & 45.1 & 46.5 & 57.8 \\
298.15 & 52.8 & 46.1 & 57 & 45.3 \\
360 & 67.2 & 57.7 & 84 & 47.0 \\
\hline \hline
\end{tabular}


TABLE VIII: Isothermal compressibility $\kappa_{T}$ for $p=1$ bar and $p=1000$ bar as obtained in this work for the TIP4P/2005 model of water. Reported values correspond to $\left(\kappa_{T} / \mathrm{bar}^{-1}\right) \times 10^{6}$.

\begin{tabular}{|c|c|c|c|}
\hline \multicolumn{2}{|c|}{$\mathrm{T} / \mathrm{K} \mathrm{p} / \mathrm{bar}$} & \multicolumn{2}{|c|}{ TIP4P/2005 Experiment } \\
\hline 260 & 1 & 51.4 & 57.8 \\
\hline 280 & 1 & 48.7 & 48.6 \\
\hline 298 & 1 & 46.3 & 45.3 \\
\hline 320 & 1 & 46.2 & 44.2 \\
\hline 340 & 1 & 47.8 & 44.9 \\
\hline 360 & 1 & 50.9 & 47.0 \\
\hline 370 & 1 & 52.3 & 48.5 \\
\hline 260 & 1000 & 42.4 & 42.3 \\
\hline 280 & 1000 & 38.5 & 37.7 \\
\hline 298 & 1000 & 37.2 & 35.7 \\
\hline 320 & 1000 & 36.7 & 34.9 \\
\hline 340 & 1000 & 36.6 & 35.1 \\
\hline 360 & 1000 & 38.2 & 36.0 \\
\hline 370 & 1000 & 39.2 & 36.7 \\
\hline
\end{tabular}



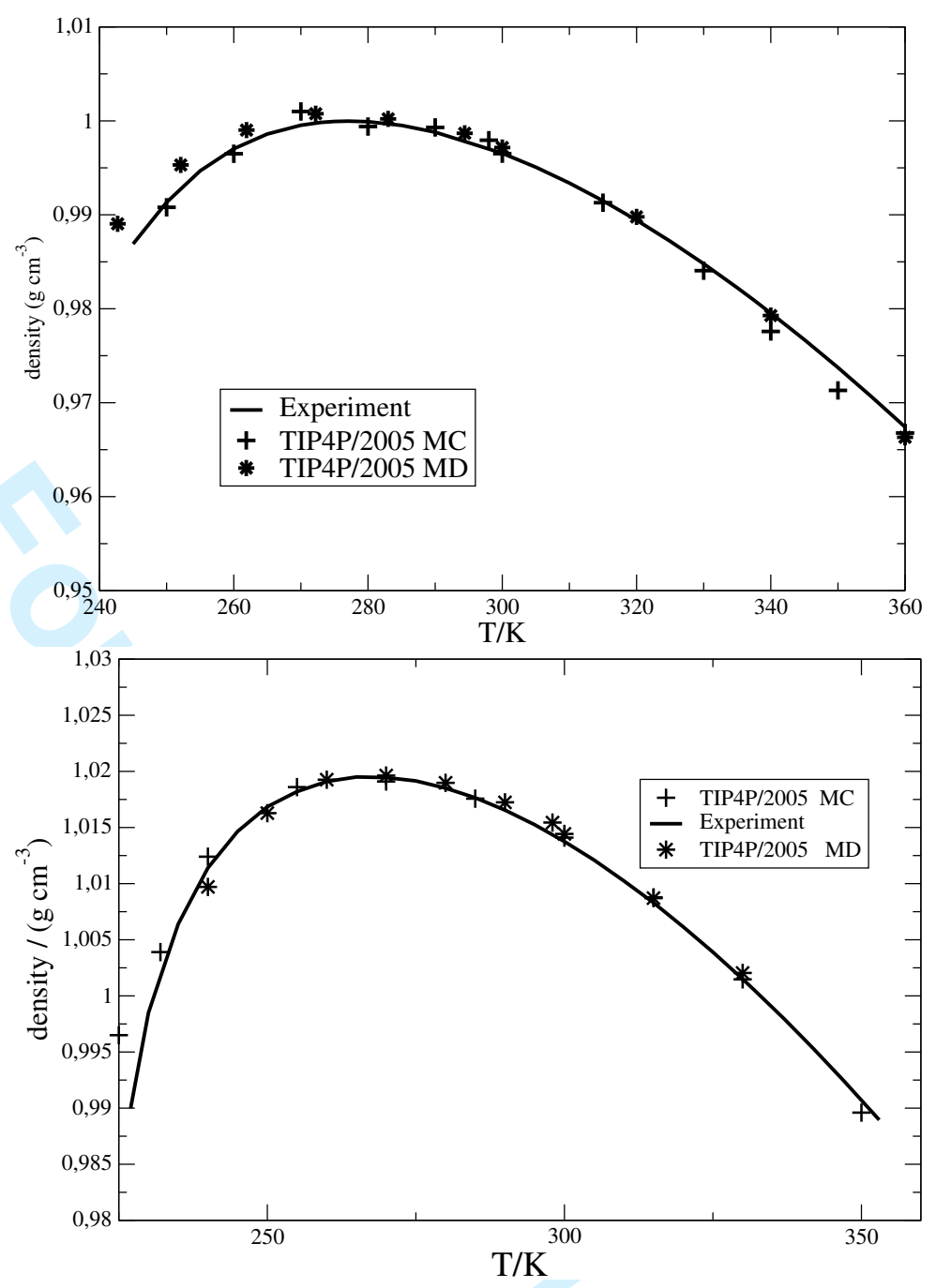

FIG. 1: Density of liquid water at $p=1$ bar (upper panel) and $p=400$ bar (lower panel) as obtained from Monte Carlo and molecular dynamics simulations with the TIP4P/2005 model. The experimental results were taken from Ref. 54 . 


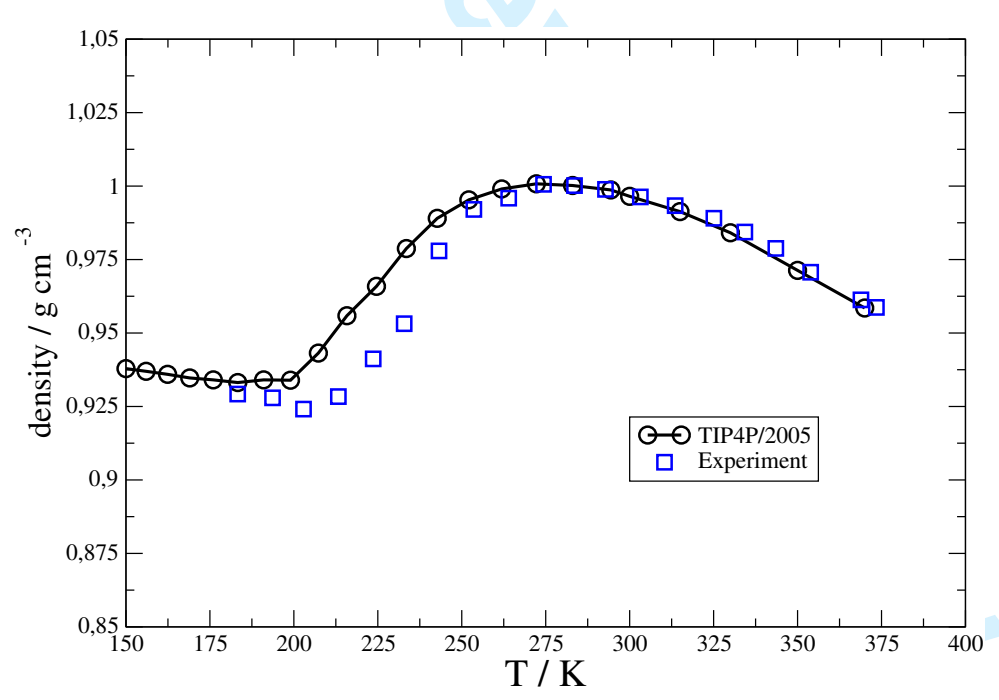

FIG. 3: Density of liquid water at atmospheric pressure as obtained from molecular dynamics simulations with the TIP4P/2005 model. For comparison, experimental data of water confined in narrow pores are also given ${ }^{48}$. 


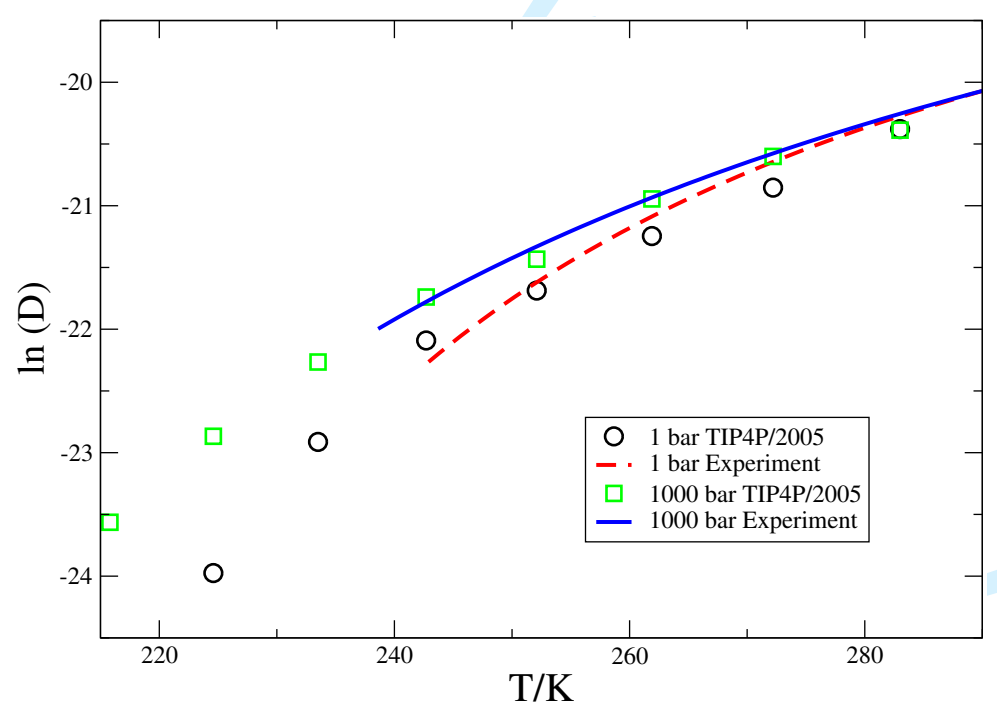

FIG. 5: Self-diffusion coefficient (in $\mathrm{cm}^{2} / \mathrm{s}$ ) of the TIP4P/2005 model at $p=1$ bar and $p=1000$ bar compared to the experimental results (taken from Ref. 53). 

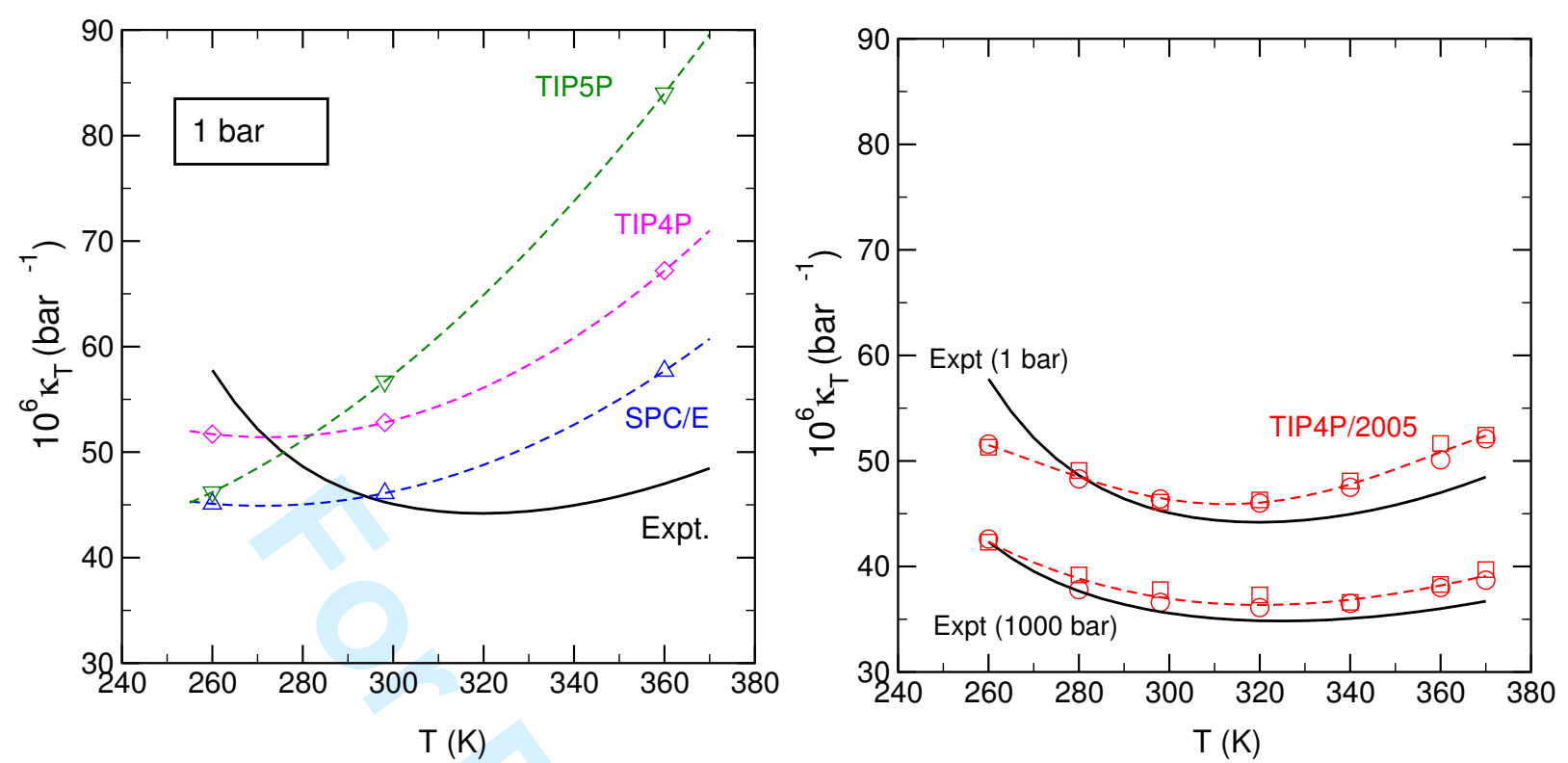

FIG. 6: Isothermal compressibility calculated from molecular dynamics simulations. Left: Results for SPC/E, TIP4P and TIP5P at $p=1$ bar using the fluctuation formula (Eq. 2). Right: Results for TIP4P/2005 using the fluctuation equation (squares) and the derivative route (Eq. 1, circles); upper results are for 1 bar and lower curves are for 1000 bar. Notice that we have deliberately used the same scale in both panels. For comparison, experimental data ${ }^{54}$ (full lines) are also plotted. 


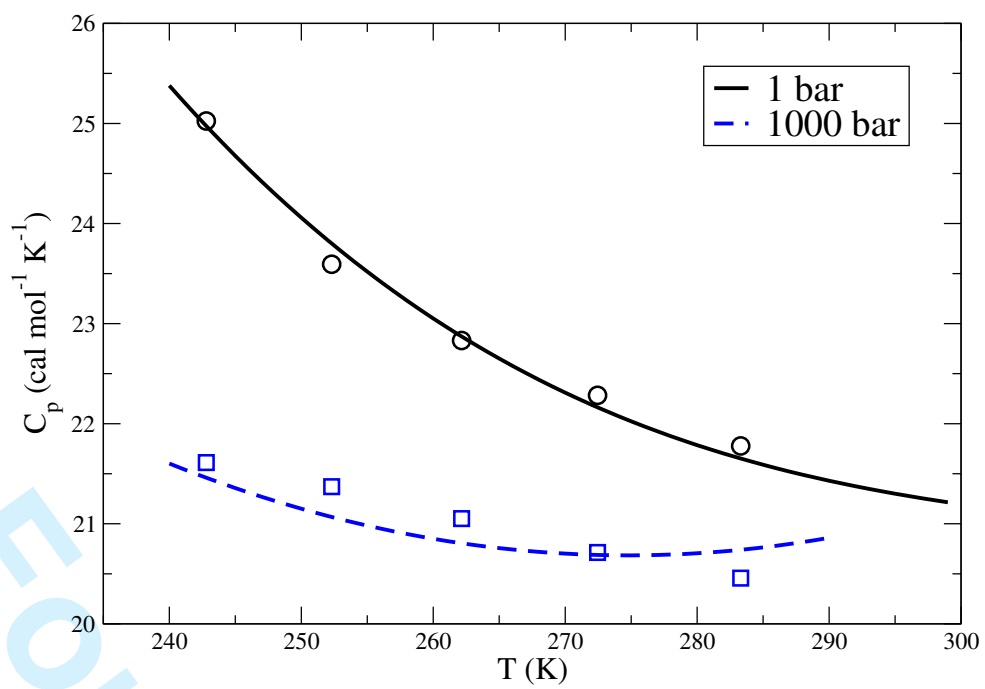

FIG. 7: Heat capacity of water at constant pressure $\left(C_{p}\right)$ as obtained from simulation results of the TIP4P/2005 for $p=1$ bar (solid line/circles) and for $p=1000$ bar (dashed line/squares). The symbols represent the results of a first degree finite difference calculation for equation (3), and the curves were obtained from the results of a third degree polynomial fit ( $\mathrm{p}=1000$ bar $)$ and fourth degree ( $\mathrm{p}=1$ bar) for the enthalpy.

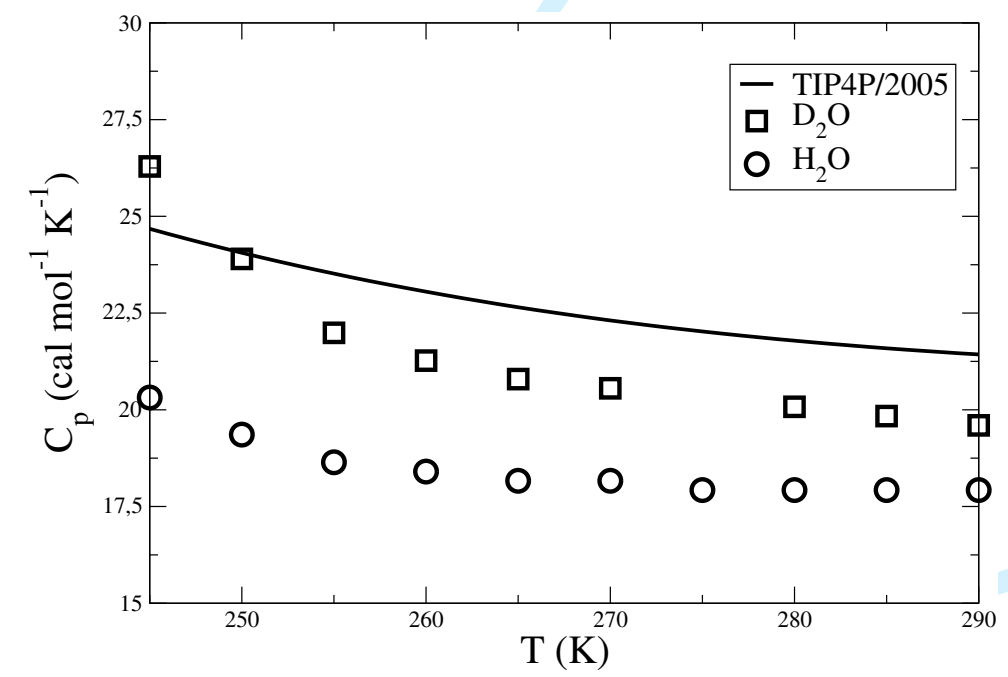

FIG. 8: Heat capacity of water at constant pressure $\left(C_{p}\right)$ as obtained from simulation results of the TIP4P $/ 2005$ for $p=1$ bar . Experimental results for $\mathrm{D}_{2} \mathrm{O}$ and for $\mathrm{H}_{2} \mathrm{O}$ taken from Angell,Oguni and Sichina ${ }^{58}$ are also presented. 
37 on 38

39

40

41

42

43

44
45

46

47

48

49

50

51

52

53

54

55

56

57

58

59

60

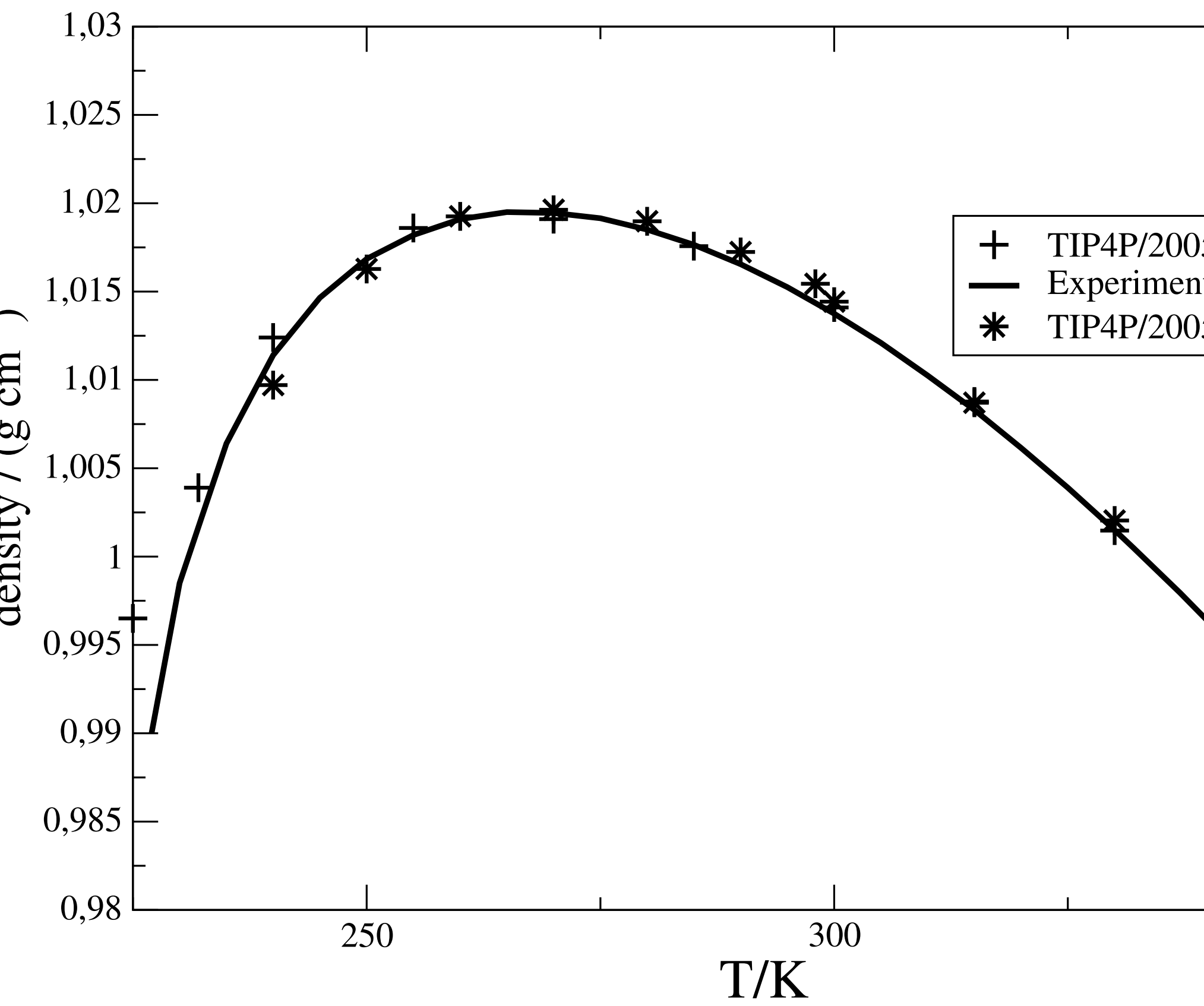

URL: http://mc.manuscriptcentral.com/tandf/tmph 


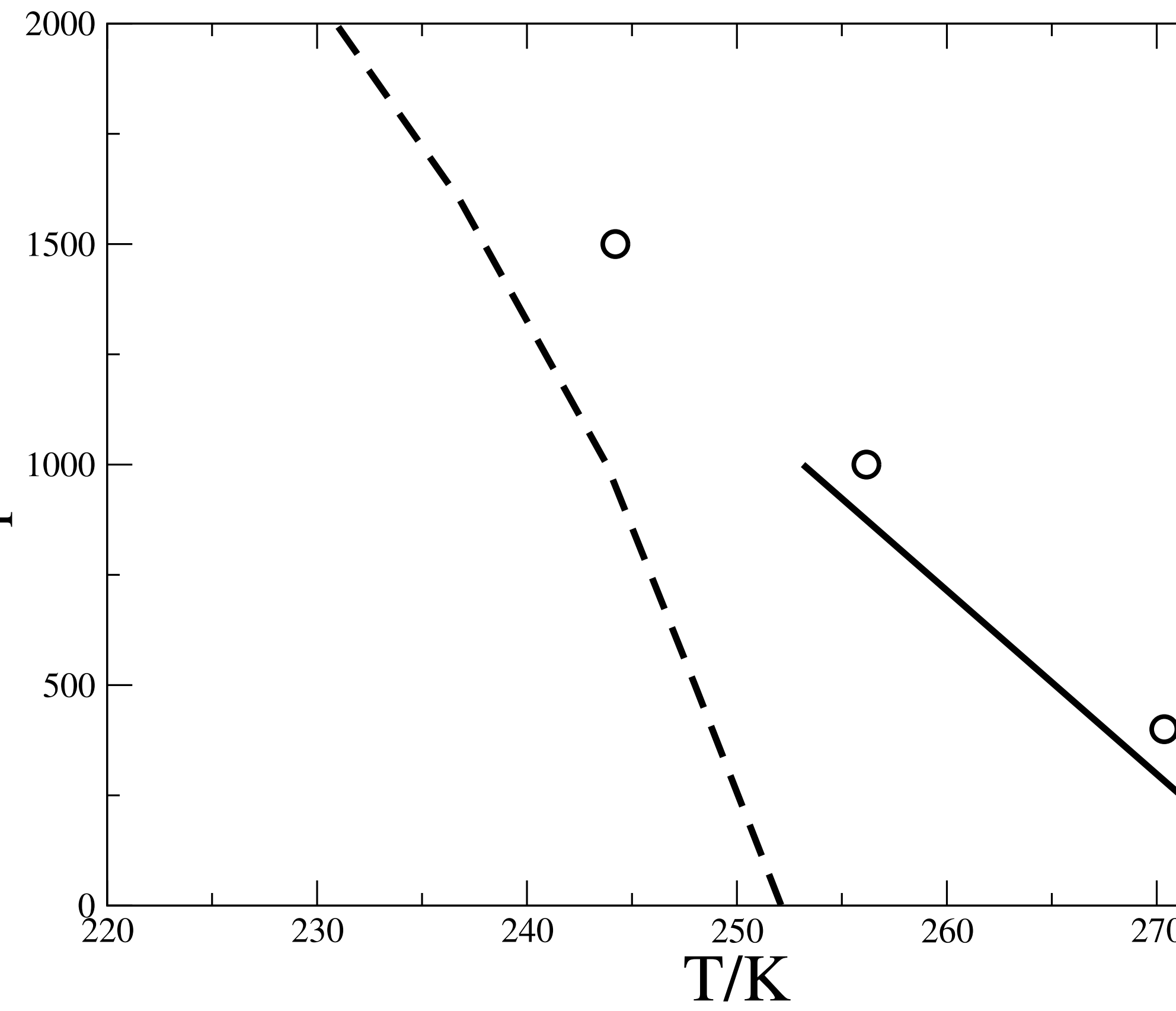

URL: http://mc.manuscriptcentral.com/tandf/tmph 


1
2
3
4
5
6
7
8
9
1
1
1
1
1
1
1
1
1
1
2
2
2
2
2
2
2
2
5
5
5
5
5
5
5
5
3
3
3
3
3
3
3
3
3
3
3
4
4

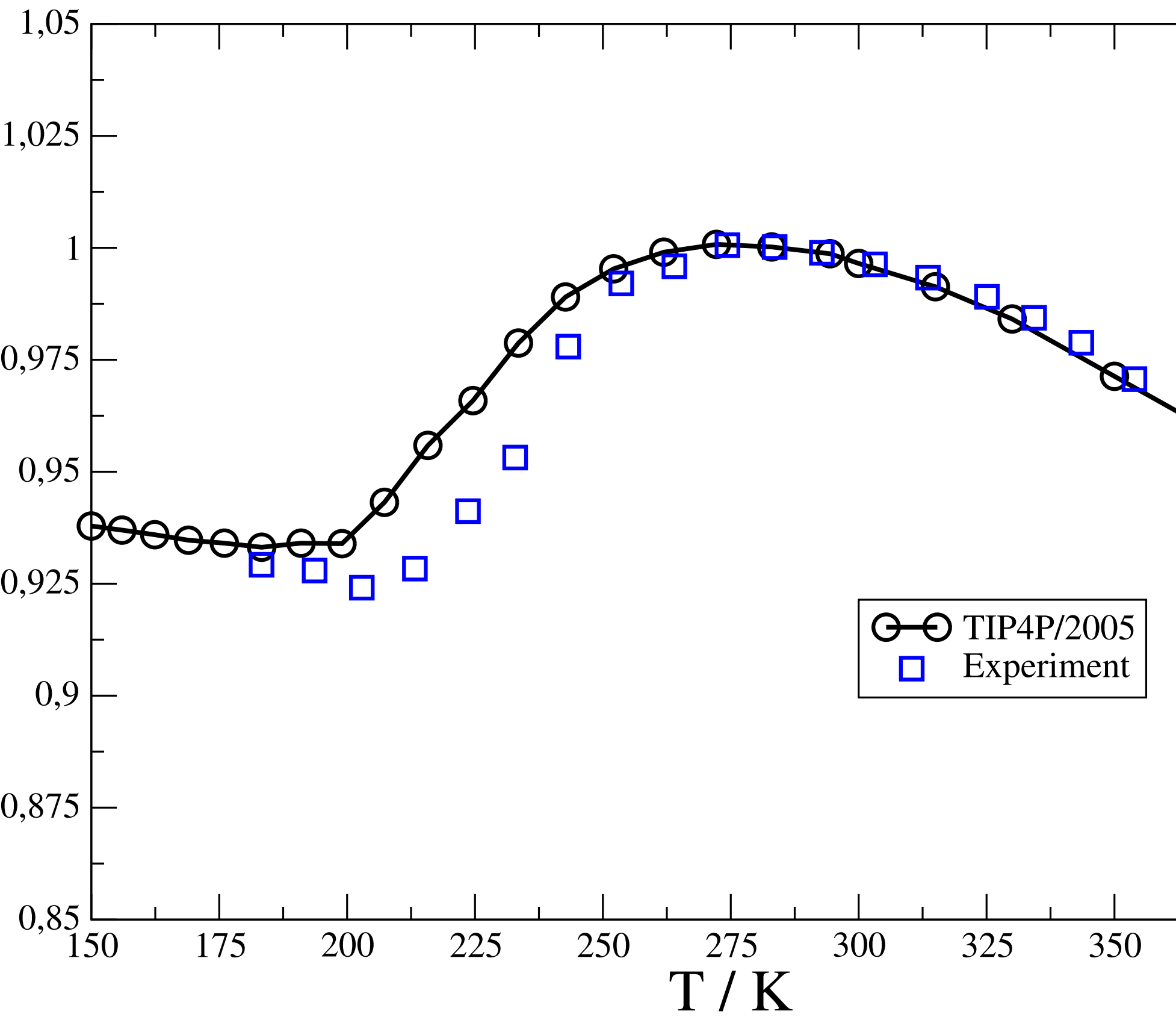

URL: http://mc.manuscriptcentral.com/tandf/tmph 


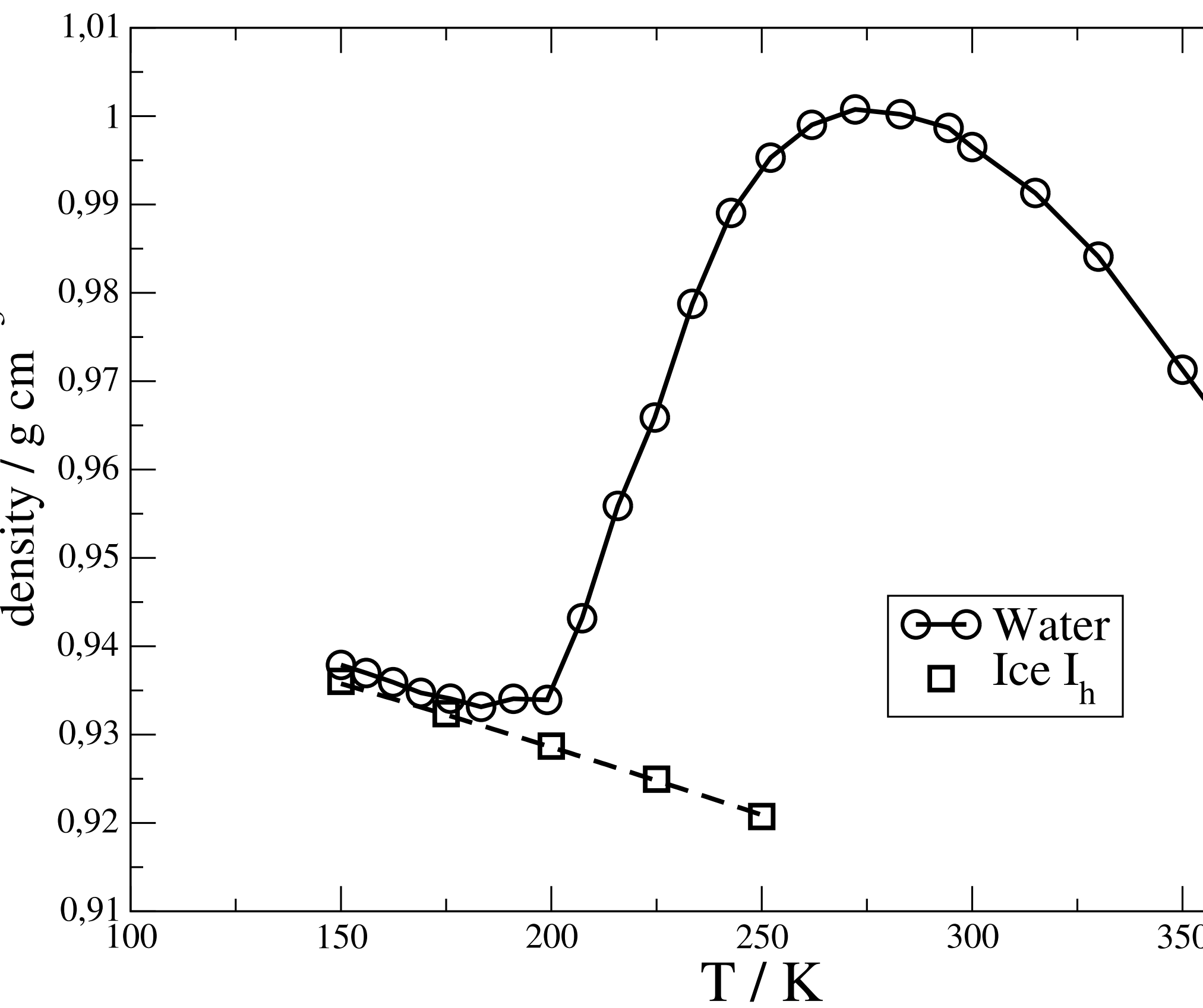

URL: http://mc.manuscriptcentral.com/tandf/tmph 


1
2
3
4
5
6
7
8
9
10
1
1
1
1
15
16
1
18
1
20
2
2
23
2
2
2

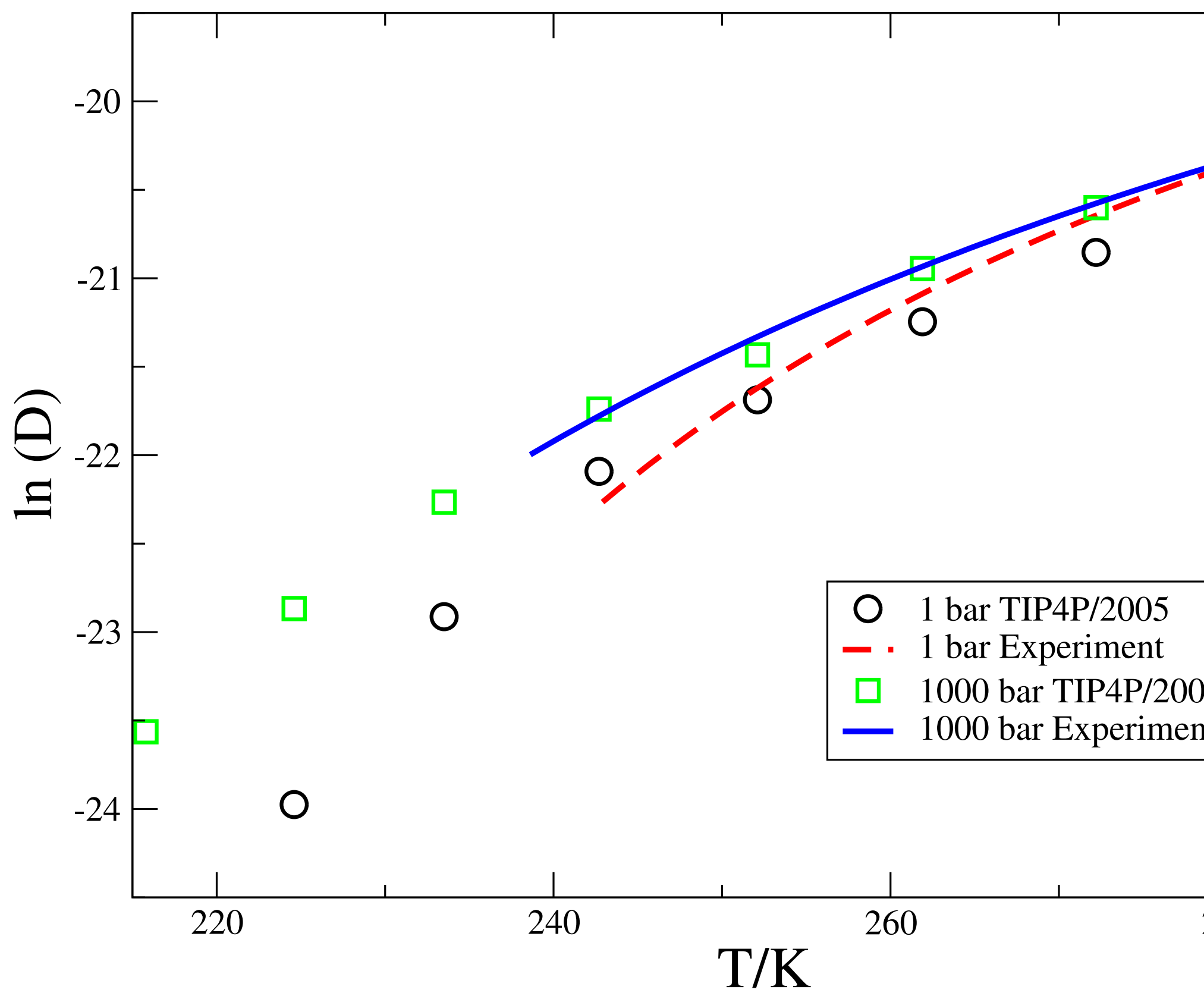

URL: http://mc.manuscriptcentral.com/tandf/tmph 


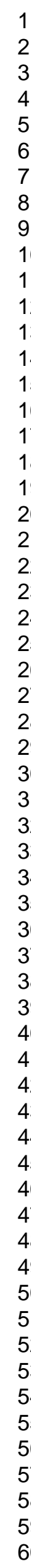

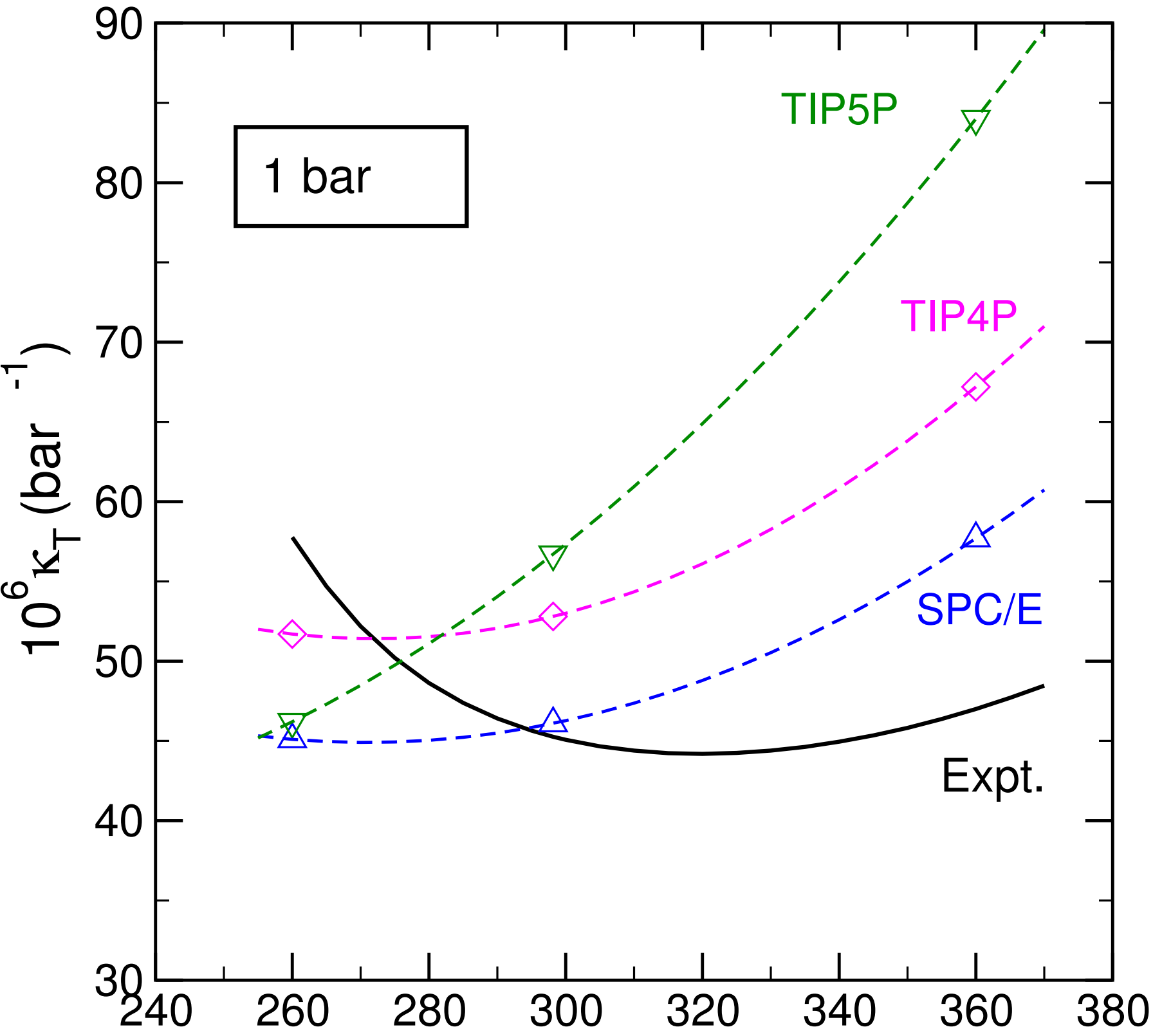

URL: http://mc.manusctip(c(Kr)al.com/tandf/tmph 


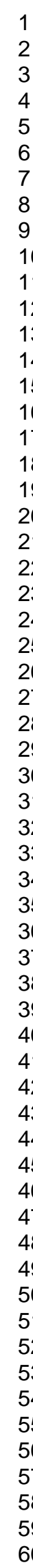

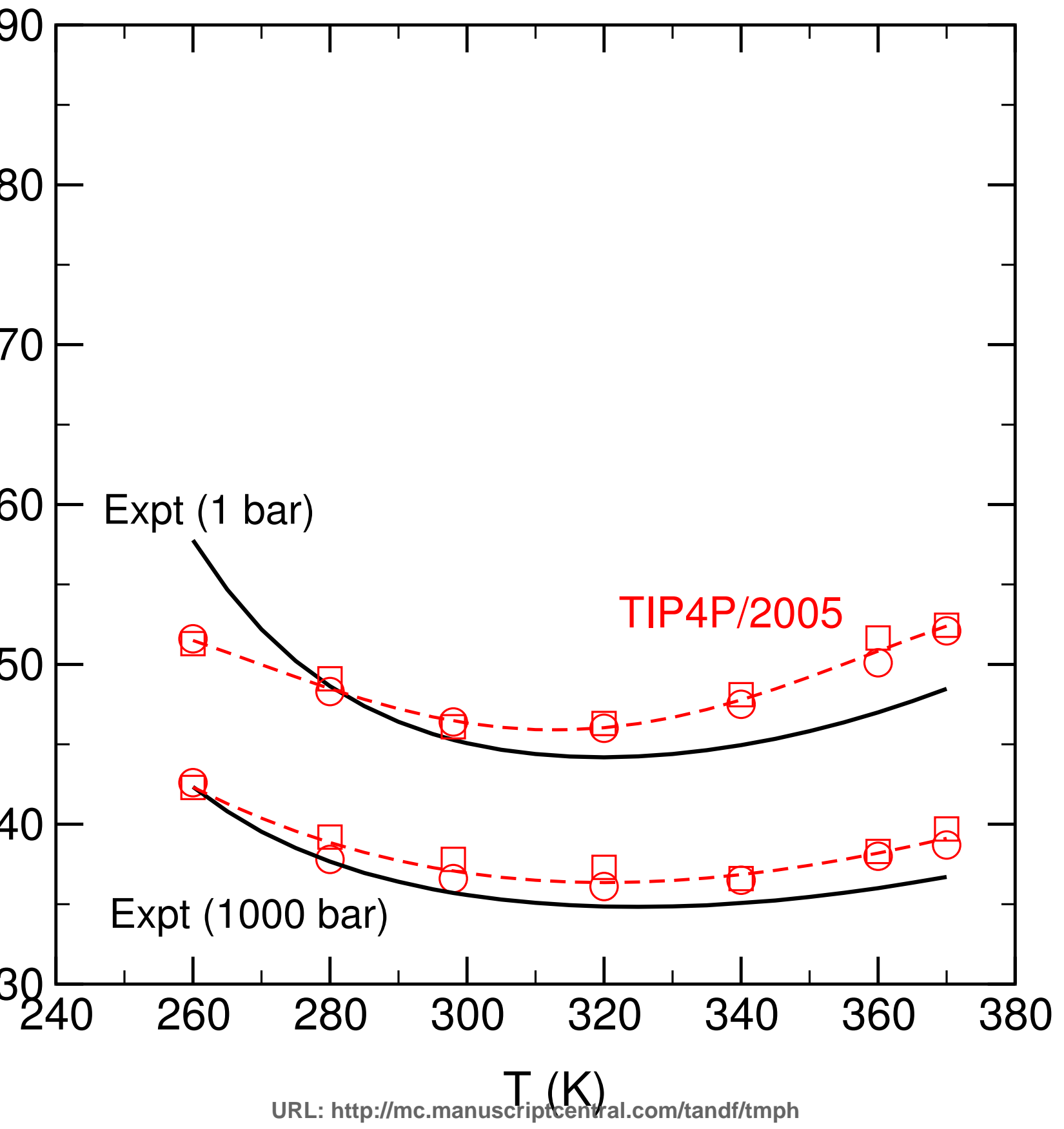




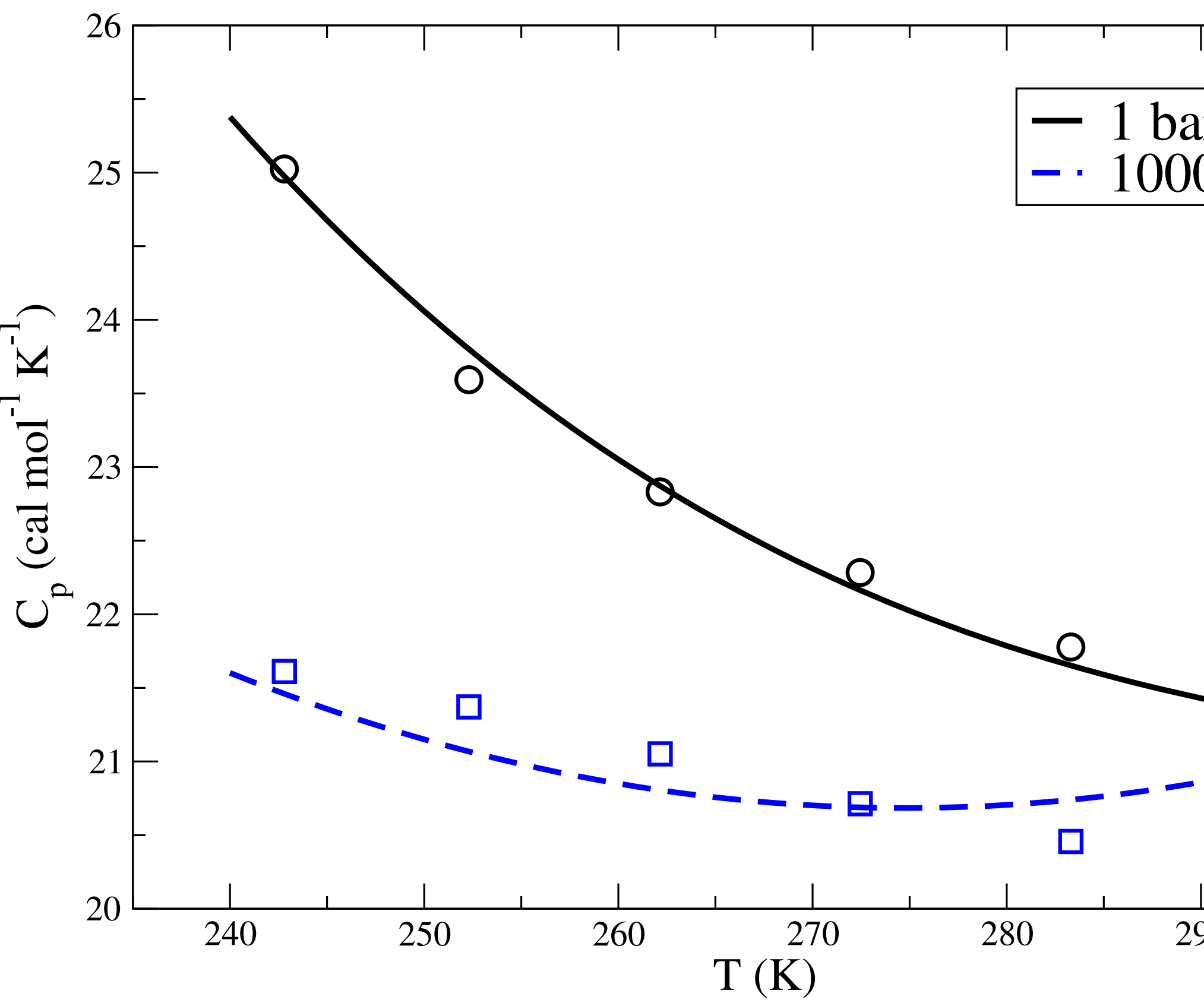

URL: http://mc.manuscriptcentral.com/tandf/tmph 


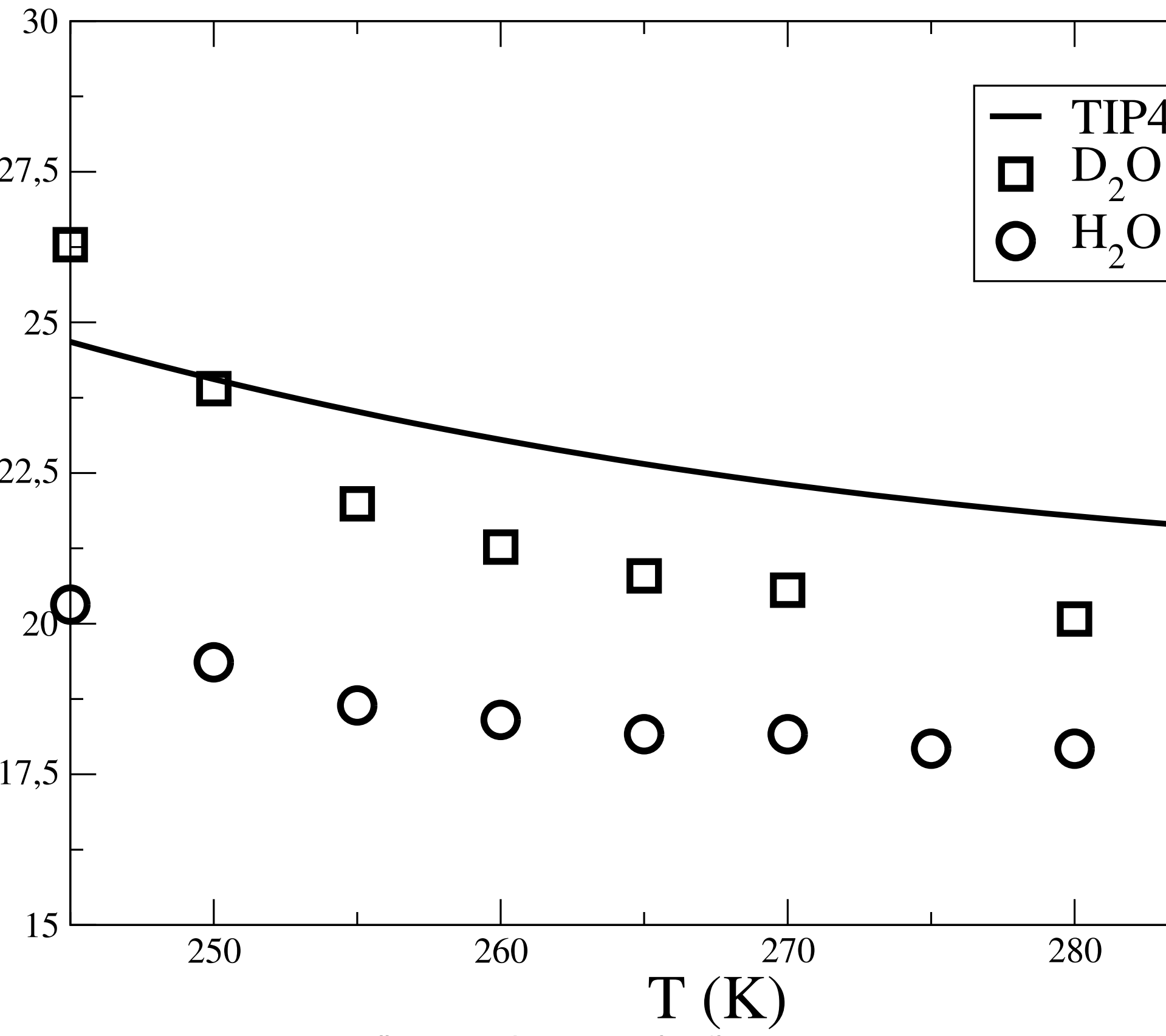

